\title{
REGISTER ZUR STUDIE
}

In diesem Register werden nur Autoren und Werke aufgelistet, die im Repertorium nicht vorkommen. Werke bekannter Autoren sind unter dem jeweiligen Verfasser aufgelistet, anonyme Schriften unter ihrem Titel. In den Repertoriumseinträgen findet sich unter der Rubrik „Studie“ der Verweis auf das Kapitel, in dem das jeweilige Werk diskutiert wird.

\author{
Abaelard \\ Collationes 112, 119, 218-219 \\ Soliloquium 163 (Anm. 3), 173-174, \\ 219 \\ Abbreviatura communitatis 153 \\ Accessus (siehe auch Konrad von \\ Hirsau) \\ zu Cicero 29 (Anm. 2), 35-36, 41 \\ (Anm. 115) \\ zu Horaz 33
}

Adam von Dryburgh, Soliloquium 20

(Anm. 103), 171, 187, 205

Adam von Perseigne 20 (Anm. 103), 171 (Anm. 33)

Adelard von Bath 68, 69

De avibus tractatus 68 (Anm. 55)

De eodem et diverso 173, 200

Quaestiones naturales 41 (Anm. 53), 68 (Anm. 55)

Adrianus Carthusiensis 195

Advocacie Nostre Dame 238

Aegidius Romanus, De triplici regimine principis 96-97

Aelred von Rievaulx, De spiritali amicitia 219

Alanus ab Insulis (Alain de Lille) 112, 114,243

Albrecht von Eyb 195

Alchemie (Textsorten) 78-79

Alexander von Lincoln siehe Dialogus inter Christianum et Fudaeum

Alkuin 69

De grammatica 39, 44, 60, 65-66

De dialectica 39, 65-66

Dialogus de rhetorica et virtutibus 39, 65-66, 98

Disputatio de vera philosophia 65-66

Disputatio Pippini 61

Liber de virtutibus et viciis $\quad 169-170$

Allegorische Epen 243

Allegorische Gedichte 158, 201
Allegorische Prosimetra siehe philosophisch-allegorische Prosimetra

Alloquia ad Spiritum Sanctum 19 (Anm.

94)

Altercatio siehe Streitgedichte, Titelgebung

Altercatio Aecclesiae contra Synagogam 117

(Anm. 41)

Altercatio duorum geometricorum 66

Altercatio Ecclesiae et Synagogae 117

Altercatio Hadriani et Epicteti 61

Altercatio inter Divinitatem et

humanitatem 52 (Anm. 125)

Altercatio inter Urbanum et Clementem

(Anm. 97)

Altercatio spiritus et carnis siehe Hildebert

von Lavardin, Liber de querimonia

Anselm von Canterbury 103, 203

Cur Deus homo 44, 216-218

De custodia interioris hominis 246

Gebete und Meditationen 170, 174, 216 (Anm. 21)

Monologion 206, 215-216

Parabeln 245-246

Proslogion 163 (Anm. 3), 215-216

Reportata (Nachschriften) 245

Anselm von Havelberg 20 (Anm. 98)

Ansprache 78, 86 (Anm. 115), 133, 164, 170, 205-206 siehe auch

Diatribe, Predigt

Antagonisms 6 (Anm. 29)

Antoninus Florentinus 50 (Anm. 110)

Apologetisch-polemische Dialoge 6-7

Apophthegmata 87

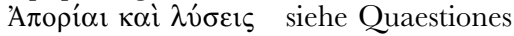
et responsiones

Arabische Literatur 78-79, 123, 253

(Anm. 15)

Argumentationstheorie 211-213

Armutsstreit 80 (Anm. 91), 139-143, 147-148, 156-157, 159, 240 
Arnold von St. Emmeram, Liber II de memoria beati Emmerami 91-92

Ars lulliana 123-128

Aucto de acusación 239

Audradus 235

Augustiner-Eremiten 241

Augustinus 8, 48-49, 203, 210, 216

Cassiciacum-Dialoge 166, 211

(Anm. 3)

Confessiones 216

De gratia et libero arbitrio 188

De vera religione 188

als Figur im Secretum 184-191

Soliloquia 163, 165-167, 169,

171-172, 174, 185-188, 207-208, 215-216, 274

Auxilius, Tractatus qui Infensor et Defensor dicitur 131, 136-137

Azzo de Correggio 194

Bachtin siehe Dialogizität

Baptista de Finario 50 (Anm. 110),

Beda 235

Beratungsgespräche $\quad 236-237,243$

Berengar von Tours 148

Scriptum contra synodum 132-133

De sacra coena 133

Bernardus Silvestris, Cosmographia 243

Bernhard von Clairvaux 171, 203

Parabolae 245-246

Soliloquium 205 (Anm. 134)

Bernhardstraktat siehe Quis dabit

Bibelexegese 62

Biographischer Dialog 5 (Anm. 25), 85, 89-90, 93-95, 215, 225-226, 270, 278

Boccaccio, Decamerone 230

Boethius, Consolatio philosophiae 9, 41, 55, 163, 167-169, 172-174, 179, 185-187, 197, 199, 201 (Anm. 120) 207-209, 210, 211 (Anm. 3), 215, 243, 274

Boso 216-217

Bridlington-Dialog 92-93

Briefdialog 254-256, 276

Bußdialog 168, 172, 181, 191

Byzantinische Literatur 48, 62-64, 78, 87, 108-109, 111 (Anm. 23), 235-236

Calcidius siehe Plato, Timaeus

Casus (Textsorte) 71 (Anm. 63)

Cassian siehe Johannes Cassianus

Chrétien de Bonneval 98 (Anm. 150)

Christian von Heiligenkreuz 241
Cicero 86, 99, 166, 185-186, 188, 214-215, 228-229

Briefe (Ad Atticum, Ad familiares) 254-255

Cato maior de senectute 35, 90 (Anm. 129), 91 (Anm. 131)

Laelius de amicitia 35, 55, 91 (Anm. 131), 186, 219

Tusculanae disputationes 167, 186, 211 (Anm. 3)

Clemens Scotus 65, 69

Collatio siehe monastische Predigt

Colloquies siehe Gesprächsbüchlein

Colloquium divine sapientie 19 (Anm. 94)

Colloquium inter sapientiam et discipulum 18 (Anm. 92)

Coluccio Salutati 254 (Anm. 22), 255

Confabulatio 97 siehe auch monastisches Gespräch

Conflictus 190-191, siehe auch

Streitgedichte

Controversia $124,252-253$

,De potestate papae'-Traktate 142-143, 159

De remediis fortuitorum siehe Seneca

Defensor von Ligugé, Liber scintillarum 169

Descensus ad inferos 234-235

Desiderius de Montecassino, Dialogus 91-92

Devotio moderna 81, 83, 195 (Anm. 106)

Dhuoda, Liber manualis 169

Dialektische Methode 111-112, 114, 132-133, 147, 213-222

Dialoganalyse $\quad 12-13$

Dialogisierte Briefe siehe Briefliteratur

Dialogisierte Exempel siehe Exempel

Dialogisierte Florilegien 196-199, 264, 275

Dialogisierte Geschichte 84-85, 225-226

Dialogisierte Protokolle 256-260

Dialogizität $\quad 14-17,280-281$

Dialogus beati Ambrosii 19 (Anm. 94)

Dialogus de musica 66-67

Dialogus de officiis divinis siehe Liber quare

Dialogus de pontificatu sanctae Romanae ecclesiae 134-135, 155 (Anm. 147), 157

Dialogus de sacris ecclesiae siehe Liber quare

Dialogus de vita vere apostolica 93, 135, 137, 145 (Anm. 126) 
Dialogus deflentis hominis et ammonentis rationis 169 (Anm. 26)

Dialogus inter Christianum et fudaeum (Alexander von Lincoln gewidmet) 111 (Anm. 23)

Dialogus inter patrem et filium 19 (Anm. 94)

Dialogus Mariae cum populo 204 (Anm. 129)

Dialogus mortis cum homine 254

Dialogus Papae et Mariae 204

Dialogus Salomonis et Marcolfi 61

Dialogus super sacra communione contra Hussitas 143 (Anm. 124)

Diatribe 54-55, 78, 79, 170 (Anm. 28), 255 siehe auch Ansprache, Predigt

Didaktische Dialoge 4, 10-11

Dirc van Herxen 195 (Anm. 106)

Disputatio 48-50, 56, 63, 146, 151, 155, 189 (Anm. 85), 239

Disputatio cuiusdam iusti cum Deo 171-172

Disputationsprotokolle 118 (Anm. 48), 256-257

Dogmatische Kontroversdialoge 130 (Anm. 86)

Dogmatischer Dialog 211-213

Dominikaner $115,140,150-153,192$, 241, 259-260

Donatus, Ars minor 60

Donatus ortigraphus 65,69

Doppeltraktat 133, 153-155, 216

Dragma 29 (Anm. 2), 38, 41, 42, 43, 58

Drama 99, 117, 131, 220, 223-224, 237-239, 246-247, 285

Eberhard von Ypern 19 (Anm. 94), 219-220, 285

Ebo von Michelsberg 89-90

Ecloga Theodoli 151

Elucidarien 10,67

Emergenter Dialog 211-213

Enea Silvio Piccolomini 50 (Anm. 112)

Ephraem Syrus 202, 236 (Anm. 6)

Eristische Dialoge 214 (Anm. 12)

Ermenrich von Ellwangen, Vita Hariolfi 91-92

Erotapokriseis siehe Quaestiones et responsiones

Errores Fudaeorum in Talmut 116

Eucherius von Lyon, Instructiones 64

Eugenius Vulgarius, Liber cuiusdam requirentis et respondentis $131,136-137$, 224 (Anm. 39), 285
Evangelium Nicodemi 234-236

Évrart d'Ypern siehe Eberhard von Ypern

Exempel 13, 80-82, 89 (Anm. 124), $110,209,225-226,244,249-251$, 253-254, 257, 260

Exorzismus 257

Florilegien 194-199, 266

Forma tradendi siehe Modus tradendi

Francesco Barbaro 227

Franziskaner 80-82, 94, 138-142, 150-153, 156-157, 192, 253

Fürstenspiegel 97-99

Galand von Reigny, Parabolae 245-246

Gaunilo 216

Gebet 166, 170, 174, 175, 201-204, 215-216

Geistererscheinung 259-260

Genera elocutionis 40-41, 43-44, 55

Geoffrey Plantagenet, Graf von Anjou und Herzog der Normandie 37, 68

Georg Alt 239

Gerhoch von Reichersberg Dialogus inter clericum saecularem et regularem 85 (Anm. 111), 136-137, 155 (Anm. 149)

Geri von Arezzo 254-255

Gervasius von Melkley, Ars poetica 30

Gespräch siehe Konversation, Mündlichkeit

Gesprächsbüchlein 10, 63-64

Gilbert Crispin 111-112, 120 (Anm. 52), 125, 217 (Anm. 25), 218, 283

Glossare 29, 42, 48, 49, 50

Glossen 28 (Anm. 2), 51 (Anm. 115), siehe auch Wilhelm von Conches

Gott (als Gesprächspartner) 176-178, 274

Gregorius Magnus, Dialogi 36-37, 41, 44, 47-48, 55, 84-88, 91, 271

Griechische Literatur 62-64, 78, 108

Großes Schisma 144-147, 157, 177

Guglielmo Alfachino 118

Gutolf von Heiligenkreuz

Dyalogus Agnetis 204

De ordine iudiciario 241

Hagiographischer Dialog 5, 85-93, siehe auch Biographischer Dialog

Handschriften

Augsburg, UB, Cod. I.2.8 19204

(Anm. 129)

Brügge, 37819 (Anm. 94) 
Cambrai, 25919 (Anm. 94)

Cambrai, 136719 (Anm. 94)

Cremona, Biblioteca Statale, 143251 (Anm. 10)

Dillingen, Studienbibliothek, XV 29241

Épinal, 5819 (Anm. 94)

Erfurt, UB, Dep. Erf., CA $4^{\circ}$ 388240

Firenze, BML, Ashburnham 1550251 (Anm. 10)

Frankfurt a. M., StUB, Ms. Praed. 59241

Frankfurt a. M., StUB, Ms. Praed. 61240 (Anm. 15), 241

Frankfurt a. M., StUB, Ms. Praed. 125241

Hildesheim, Dombibliothek, J30 204 (Anm. 130)

Innsbruck, UB, 33, 34, 131, 216, 481, 58418 (Anm. 92)

Leipzig, UB, Haen. 15240 (Anm. 15)

Lilienfeld, StiftsB, 137241 (Anm. 19)

London, BL, Royal 6. D. X 146

Lübeck, Bibliothek der Hansestadt, Cod. theol. lat. 64 240-241

Mainz, StB, I, 151240 (Anm. 15)

Marseille, BM, 211240 (Anm. 15)

München, BSB, Cgm 660240 (Anm. 15)

München, BSB, Clm 1222251 (Anm. 10)

München, BSB, Clm 2889143 (Anm. 124)

München, BSB, Clm 3331240

München, BSB, Clm 3334240 (Anm. 15), 241

München, BSB, Clm $3547 \quad 198$

München, BSB, Clm 6499198

München, BSB, Clm 14831241

München, BSB, Clm 1863897

München, BSB, Clm 19475 41, Anm. 52

München, BSB, Clm $27421 \quad$ 240, 241

Oxford, BodL, Bodley $52 \quad 240$

Padova, BU, 150918 (Anm. 92)

Paris, BnF, lat. 8507251 (Anm. 10)

Paris, BnF, lat. 18216240 (Anm. 15)

Soissons, 22419 (Anm. 94)

Toledo, Archivo y Biblioteca Capitular, 10-28 251 (Anm. 10)

Torino, BNU, H. III. 6251 (Anm. 10)
Uppsala, UB, C $17 \quad 143$ (Anm. 124)

Uppsala, UB, C 131240 (Anm. 15)

Vaticano, BAV, Vat. lat. 10499235

(Anm. 2)

Wien, ÖNB, 362241

Würzburg, UB, M.p.th.f. 55240

(Anm. 15)

Heinrich II., König von England 37, 68, 98-99

Heinrich von Augsburg 67

Heinrich von Mügeln, De artibus 200

Heinrich von Settimello, Elegia 53, 201

Herbord von Michelsberg, Vita Ottonis episcopi Babenbergensis 89-90

Hildebert von Lavardin, Liber de querimonia 19 (Anm. 94), 172-174, 201 (Anm. 120), 244, 264

Hildegard von Bingen 257

Historische Dialogforschung 12-13

Hof (als literarisches Zentrum) 37, 39, 65-66, 68, 69, 71, 77 (Anm. 80), 159, 161-162, 227, 247, 277-280

Honorius Augustodunensis 67 (Anm. 43), 85 (Anm. 111), 93 (Anm. 137)

Clavis physicae 40

De esu volatilium 92-93

Elucidarium 19 (Anm. 94), 67-68, 71

Hraban siehe Rabanus Maurus

Hugo Metellus, Certamen regis cum papa 134 (Anm. 97)

Hugo von St. Victor 85 (Anm. 111), 179

Adnotationes elucidatoriae in quosdam

Psalmos David 237

De grammatica 67

De sacramentis 67

De vanitate mundi 171

Dialogus de sacramentis legis naturalis et scriptae 67

Didascalicon 33, 67 (Anm. 44)

Epitome Dindimi in philosophiam 67

Libellus de formatione arche 48

Soliloquium de arrha animae 170-171, $175,205,244$

Hugo von Vaucemain 178

(Anm. 47)

Humanismus 64, 70-71, 101, 184-196, 222-227, 231-232, 254-256

Hussiten 143 (Anm. 124)

Idung von Prüfening 135-136, 155 (Anm. 149) 
Inkunabeln

HC $15236 \quad 198$

Inquisition 115-121, 128-129, 138, 256,279

Interreligiöse Dialoge 7

Interrogationes et responsiones 43-45, siehe auch Quaestiones et responsiones

Investiturstreit 133-135

Ioca monachorum 61

Isidor von Sevilla

De vetere et novo testamento quaestiones 64 Etymologiae 30, 31-34, 40, 44, 47, 48, 50-51, 54, 56, 58, 204-205 Synonyma 9, 19 (Anm. 94), 163-164, 168-174, 191, 207-208, 271

Islam 112-113, 160, 217-218

Iunilius Africanus, Instituta regularia divinae legis 64

Jacobus de Theramo

Belial 19 (Anm. 94), 100, 239-240, 264

De monarchia mundi ecclesiastica et temporali (Monarchialis) 54

Jakob I., König von Aragón 121

Jakob van Maerlant 239

Jakob von Voragine, Legenda aurea 235

Joachim von Fiore 140

Johann von Neumarkt 206 (Anm. 137)

Johannes Balbi, Catholicon 39, 42, 43-44, 47, 48

Johannes Burgundi 158 (Anm. 150)

Johannes Cassianus, Collationes 85-88, 103, 178 (Anm. 51)

Johannes von Fécamp 170, 174, 201, 216 (Anm. 18)

Johannes Fogel 240-241

Johannes Gerson, Consolatio Philosophiae 200

Johannes Kautsch 82 (Anm. 98)

Johannes de Lignano 144, 153

Johannes Scottus Eriugena, Periphyseon 145 (Anm. 126), 215

John Barton, Altercatio lollardi cum catholico 143 (Anm. 124)

John Maynsforth 240

John Wyclif 105, 148, siehe auch Lollarden

Joseph ben Nathan Official, Vikuah Rabbenu Yehiel mi-Paris 256-257

Juan Manuel, Infante, Liber de los enxiemplos 250
Judentum 104-105, 107-121, 217-218, 272-273, 279, 283-284

Kanoniker 92-93, 135-137, 195

Karl V., König von Frankreich 154

Karl der Große 39, 65-66, 69, 70

Karmeliter 138-139, 150-153

Kartäuser 80, 83 (Anm. 102), 171

(Anm. 33), 195, 241

Katechismen 4, 10

Katharer 112, 114, 138

Katharina von Siena 183 (Anm. 64)

Kleanthes 193 (Anm. 97)

Kluniazenser 135-136

Konrad von Hirsau,

Dialogus de mundi contemptu vel amore 135, 155 (Anm. 149)

Dialogus super auctores $35-37,47,50$, 53-54, 56

Konrad von Megenberg, Planctus ecclesie in Germania 53, 158 (Anm. 150)

Kontrovers- und Bekehrungsgespräch 6 (Anm. 29)

Konversation 12-14, 99 (Anm. 153), 166 (Anm. 9), 222-225, 229

Kritische Dialoge 214 (Anm. 12)

Lanfranc, Tractatus de corpore et sanguine Domini 132-133, 136, 153, 155

Lefèvre d'Étaples 127 (Anm. 77)

Lehrer-Schüler-Dialog 4

Lehrgespräch 4, 12-13, 59, 243

Liber de secretis naturae 78

Liber dictus peccatorum consolatio siehe Jacobus de Theramo, Belial

Liber exceptionum ex libris viginti trium auctorum 195 (Anm. 107)

Liber quare 19 (Anm. 94), 62 (Anm. 19)

Liutprand von Cremona, Relatio de legatione Constantinopolitana 48

Lollarden 143 (Anm. 124), 159 siehe auch John Wyclif

Lovato Lovati 70-73, 224

Lucidarien 10, siehe auch Elucidarien

Lukianischer Dialog 22

Lullismus 127

Lullscher Dialog 127-130, 273-274

Malcalanus, Dialogus de statu ecclesiae 132, 155 (Anm. 147)

Marguerite Porete, Mirouer 183 (Anm. 64)

Marienklagen 53, 202-204, 209, 266, 275 
Marsilius von Padua 71

Martianus Capella 172, 174, 199, 243

Martin von Leibitz 50 (Anm. 110)

Mechthild von Magdeburg, Fließendes Licht der Gottheit 183

Meditation 170, 174, 187, 193, 201-207, 215-216, 219, 244

Memorandum 266

Mendikanten 82, 118, 121, 138-139, 143, 148, 150-153, 240 siehe auch Augustiner-Eremiten, Dominikaner, Franziskaner, Karmeliter

Modus dialogi 46-47, 129

Monastische Beichte 84

Monastische Literatur 83, 89 (Anm. 124)

Monastische Predigt 84, 245

Monastische Streitgespräche 7, 92-95, 135-136, 271

Monastisches Gespräch 37, 47, 84, 97,245

Moralphilosophische Traktate 169-170, 194, 275

Mystère de l'Advocacie 239

Nicolaus von Gues 50 (Anm. 110), 127 (Anm. 77)

Nicolaus Jacquier, Dialogus de sacra communione 143 (Anm. 124)

Nikolaus von Dresden, De purgatorio 143 (Anm. 124)

Nikolaus von Jauer, Dialogus super sacra communione 143 (Anm. 124)

Nikolaus von Thüringen 18 (Anm. 92)

Odo von Cambrai, Disputatio contra Iudaeum Leonem nomine 44, 109 (Anm. 17), 111 (Anm. 23), 218, 283-284

Odo von Cluny (?) siehe Dialogus de musica

Ostkirchen 123-124, 147, 149

Otloh von St. Emmeram, De mysteriis numeri ternarii 66 (Anm. 41)

Papias, Elementarium 29 (Anm. 2), 33

Papst (Machtbefugnisse) 142-145, 149, 159

Parabeln 245-246, 250

Paschalis Romanus 109, 120

Paschasius Radbertus, Epitaphium Arsenii 89-90

Patarener siehe Katharer

Paulus Burgensis (von Burgos), Scruptinium scriturarum 39-40, 50, 56
Pentalogus 50-51

Perceval Spinola 99

Petrarca, Briefe 255

Petrus Abaelardus siehe Abaelard

Petrus Alfonsi

Dialogus 112-113, 119, 120 (Anm. 52), 174, 212

Disciplina clericalis 250 (Anm. 6)

Petrus von Blois, Dialogus inter Henricum regem et abbatem Bonnevallis 98-99

Petrus Cantor 95-96, 98-99

Petrus Damiani Antilogus 109 (Anm. 17) Disceptatio sinodalis 134, 136, 155 (Anm. 147)

Petrus Helias, Summa super Priscianum 42

Petrus de Luna 154, 157

Petrus de Vinea 253

Pharetra fidei (Pharetra-Traktat) 116

Philipp der Kanzler, Dialogus Virginis cum cruce 203-204

Philipp der Schöne, König von Frankreich 95-96, 99, 142-143

Philosophisch-allegorische Prosimetra 172-174, 199-201, 208-209, 223, 243

Piaito ch'ebbe Dio con l'inimico 234-235

Planctus ante nescia 202

Planctus Mariae siehe Marienklagen

Plato, Timaeus 38, 41-42, 55

Plinius der Jüngere, Briefe 254-255

Poetiklehren 30

Polyperspektivik 16, 280

Prager Reformbewegung 82-83

Prämonstratenser 135-137, 150 (Anm. 37)

Predigt $33,34,37,48,55,81-82$, 245-246, 249-251, 266-267

Prosimetrum siehe philosophischallegorische Prosimetra

Protokolle 63 (Anm. 24), 256-260, siehe auch Disputationsprotokolle, Visitationsakten, Zeugenaussagen

Prudentius 235, 243

Ps. Raymundus Lullus, Liber de secretis naturae 78

Quaestio (Textsorte) 10, 63, 67-68, 72 Quaestiones de iuris subtilitatibus 68 (Anm. 51)

Quaestiones et responsiones 5, 58, 62-63, 72, 73, 103, 111, 114, siehe auch Interrogationes et responsiones Quis dabit 53, 202-203 
Rabanus Maurus, De computo 66, 69

Raschi 114

Rather von Verona, Dialogus confessionalis 54-55, 170 (Anm. 28)

Raymundus de Rocosello, Certamen anime 245 (Anm. 8)

Reciprocatio 171 (Anm. 35)

Religionsdialoge 6-7

Religionsgespräche 6-7

Renaissance-Dialog 14

Renaut de Louhans 192 (Anm. 95)

Richard FitzNigel (von Ely), Dialogus de scaccario 68, 69 (Anm. 57)

Richard Löwenherz, König von England 98

Richard Rufus, Speculum animae 222 (Anm. 37)

Richard von St. Victor, $D e$ Emmanuele 40

Robert von Chester siehe Testamentum Morieni

Rodrigo Jiménez de Rada, Dialogus libri vite 54

Rodulfus monachus 170 (Anm. 29)

Rogerius Enodationes quaestionum super codice 68 Quaestiones super Institutis 68

Rudolf von St. Trond, De virginitate Mariae 53

Rupert von Deutz 85 (Anm. 111), 93 (Anm. 137), 119 Altercatio monachi et clerici 135-137, 155

Anulus 109 (Anm. 17)

siehe auch Dialogus de vita vere apostolica

Salonius von Vienne 64 (Anm. 30)

Scholastische Dialoge 10-11

Schulliteratur 10-11, 34, 39, 58, 59-64, 70-72, 103, 195-196, 253, $266,277-280$

Seneca, 194 (Anm. 102), 195, 198 De remediis fortuitorum 101, 163 (Anm. 2), 192-193, 274

Dialogi 54

Epistolae ad Lucilium 255-256

Tragödien 70-73, 131, 223-224, 285

Sermo 32-33, 36

Simon Batz von Homburg 240-241

Soliloquia animae ad Deum 19 (Anm. 94), 205-207

Soliloquium 9, 19 (Anm. 94), 163-166, 204-207, 216 (Anm. 17)
Soliloquium quod habuit Jesus cum Maria matre sua 205

Speculum virginum $\quad 92-93$

Stephan von Olmütz, Dialogus volatilis inter aucam et passerem 143 (Anm. 124)

Streit der Töchter Gottes 236-237, 243-245

Streitgedichte 3 (Anm. 10), 51-53, 117 (Anm. 40), 127 (Anm. 76), 134,155, 239, 244-245, 252-254, 266

Sulpicius Severus 85-90, 230, 271

Syrische Literatur 202, 236

Talentproben 158

Talmud 114, 115, 116, 118, 120, 160

Testamentum Morieni 79

Tetralogus $\quad 50-51$

Teufel 233-241, 257-260, 275-276

Thaddäus von Suessa 253

Theater siehe Drama

Theoderich von Chartres, Heptateuchon 70 (Anm. 61)

Thomas von Aquin, 140 De regimine regni ad regem Cypri 96-97

Thomas von Chobham 206

Titelgebung 30, 50-55, 136 (Anm. 107), 163-164, 171, 190-191, 203-207, 218 (Anm. 31), 239

Tra(c)tado 30 (Anm. 4)

Tractatus 30 (Anm. 4), 32-33

Tractatus Micreris 79

Trialogus $\quad 50-51$

Trinität (Beratung) 236-237, 242-244

Trostbücher 9-10, 100-101

Tröstungsdialoge 10

Turba philosophorum 79

Übersetzungen in die Volkssprachen siehe Volkssprachen

Latinisierungen $38,41-42,63,79$, 87, 109, 183 (Anm. 64)

Ugo di San Severino 95

Ulrich Tenngler, Layen Spiegel 239

Universitäre Debatten 158-159

Universitäre Textsorten $\quad 67-68,69,71$, 158-159, 195-196, 222, 230-232, 279 siehe auch Casus, Disputatio, Quaestio

Universitäten $\quad 63,67-69,71-72,102$, 105, 115, 139-140, 159, 161-162, 195-196, 222, 227, 230-231, 240-241, 247, 252-253, 279

Unterweisungsgespräch 4 
Varro, Bimarcus 163 (Anm. 2)

Vinzenz von Beauvais, Speculum historiale 235

Visio Philiberti 244

Vision 148-149, 154, 172-173, 179-181, 187, 200, 203, 218

Visitationsakten 256

Vita beatae virginis Mariae et Salvatoris rhythmica 205

Vocabularius ex quo 33, 47

Volkssprachen 10 (Anm. 51), 29 (Anm. 2), 48, 51, 61, 67, 81, 83, 117, 124 (Anm. 67), 125, 170 (Anm. 31), 192, 195-196, 209, 236 (Anm. 7), 238-239, 244 (Anm. 7), 251, 260 Katalanisch 48, 124 (Anm. 67), 249

Kastilisch 48, 61, 198, 239, 250

Deutsch 61, 117, 169, 206, 239-240

Englisch 48, 61, 143 (Anm. 124), $159,169,206$

Französisch 48, 61, 117, 169 (Anm. 26), 192 (Anm. 95), 206, 238-239, 257

Italienisch 206, 230, 234-235

Isländisch 48

Niederländisch $\quad 83,198,239$

Portugiesisch 48, 206

Slawisch (Alt-, Kirchen-) 235-236

Vulgarius siehe Eugenius Vulgarius

Waldenser 108, 138

Warnerius von Basel, Paraclitus 53, 172

Weltklerus 71, 80, 82, 135-136, 140, 150-153, 159, 192, 279-280
Wernher von Regensburg, Liber

soliloquiorum 206

Wiclyf siehe John Wyclif

Wido von Ferrara, De scismate Hildebrandi 134, 137, 155 (Anm. 147), 157

Wilhelm von Aragón 39, 56

Wilhelm von Bourges 110 (Anm. 18)

Wilhelm von Conches 37, 48, 54, 56, 58, 69

Dragmaticon 37, 39, 68, 69 (Anm. 57), 98, 228

Glosae super Boethium 37-42

Glosae super Platonem 38-42

Glosae super Priscianum 37-42

Wilhelm von Hirsau 66

Wilhelm von Ockham, Opus nonaginta dierum 153

Wilhelm von Sarzano 158 (Anm. 150)

Wilhelm von St. Denis 90

Willelm Jordaens, Conflictus virtutum et vitiorum 245 (Anm. 8)

William de Montibus, Similitudinarium 245-246

Wipo, Tetralogus 50 (Anm. 111)

Wisdom and curiosity dialogues siehe Witzdialoge

Witzdialoge 61, 271

Zetemata siehe Quaestiones et responsiones

Zeugenaussagen 81 (Anm. 93), 256

Zisterzienser 80-83, 135-136, 150

(Anm. 37), 195, 241, 245

Zwiegespräch 50-51, 56 